\title{
Mo and W bis-MGD enzymes: nitrate reductases and formate dehydrogenases
}

Received: 10 May 2004 / Accepted: 14 June 2004 / Published online: 12 August 2004

(c) SBIC 2004

\begin{abstract}
Molybdenum and tungsten are second- and third-row transition elements, respectively, which are found in a mononuclear form in the active site of a diverse group of enzymes that generally catalyze oxygen atom transfer reactions. Mononuclear Mo-containing enzymes have been classified into three families: xanthine oxidase, DMSO reductase, and sulfite oxidase. The proteins of the DMSO reductase family present the widest diversity of properties among its members and our knowledge about this family was greatly broadened by the study of the enzymes nitrate reductase and formate dehydrogenase, obtained from different sources. We discuss in this review the information of the better characterized examples of these two types of Mo enzymes and $\mathrm{W}$ enzymes closely related to the members of the DMSO reductase family. We briefly summarize, also, the few cases reported so far for enzymes that can function either with Mo or $\mathrm{W}$ at their active site.
\end{abstract}

Keywords DMSO reductase family $\cdot$ Formate dehydrogenase $\cdot$ Molybdenum-containing enzymes ·

Nitrate reductase $\cdot$ Tungsten-containing enzymes

Abbreviations AOR: aldehyde oxidoreductase - DMSO: dimethyl sulfoxide $\cdot$ EXAFS: extended X-ray absorption fine structure $\cdot \mathrm{Fdh}$ : formate

J. J. G. Moura $(\bowtie) \cdot$ C. D. Brondino $(\bowtie) \cdot$ J. Trincão

M. J. Romão

REQUIMTE/CQFB, Departamento de Química, Faculdade de Ciências e Tecnologia, Universidade Nova de Lisboa, 2829-516

Caparica, Portugal

E-mail: jose.moura@dq.fct.unl.pt

Tel.: + 351-21-2948382

Fax: + 351-21-2948550

E-mail: carlos.brondino@dq.fct.unl.pt

Tel.: + 54-342-4575213

C. D. Brondino

Facultad de Bioquímica y Ciencias Biológicas,

Universidad Nacional del Litoral,

3000 Santa Fe, Argentina dehydrogenase $\cdot$ Fdh-H: formate dehydrogenase component of the formate-hydrogen lyase complex of E. coli $\cdot$ Fdh-N: nitrate-dependent formate dehydrogenase of $E$. coli when growing anaerobically on nitrate MGD: pterin cofactor with a guanine dinucleotide $\cdot$ Nap: periplasmic nitrate reductase $\cdot$ Nar: respiratory nitrate reductase $\cdot \mathrm{SRB}$ : sulfate reducing bacteria TMAO: trimethylamine $N$-oxide

\section{Mononuclear Mo- and W-containing enzymes: general properties and classification}

Molybdenum and tungsten are second- and third-row transition elements, respectively, which are found in a mononuclear form in the active site of a diverse group of enzymes that generally catalyze oxygen atom transfer reactions $[1,2]$. Despite the high similarity between the chemical properties of $\mathrm{Mo}$ and $\mathrm{W}, \mathrm{W}$-containing enzymes represent only a small percentage compared to Mo-containing enzymes. Mo-containing enzymes are found in almost all forms of life [1], whereas W-containing enzymes seem to be exclusive for organisms such as hyperthermofilic archaea that live in extreme environments [2]. However, W-containing enzymes have been found in organisms that do not need extreme conditions $[3,4,5]$, suggesting a more important role for tungsten [6]. Some of the mononuclear W-containing enzymes described present active sites similar to the Mo enzymes, but they have different 3D structures and have been classified in a different group [2,7]. However, as we discuss below, other $\mathrm{W}$ enzymes are structurally closely related to the Mo enzymes, and are the ones included in this review.

Mononuclear Mo-containing enzymes have been divided into three groups called the xanthine oxidase, dimethyl sulfoxide (DMSO) reductase, and sulfite oxidase families [1]; the active site of these three families can be represented as shown in Fig. 1 [1, 8]. The metal atom is coordinated by the thiolates of the 
Fig. 1 Top: active-site structure of the three families of mononuclear and Mo and W enzymes. Bottom: active site of the enzymes formate dehydrogenase and nitrate reductase belonging to the DMSO reductase family. $\mathrm{X}$ and $\mathrm{Y}$ represent ligands such as oxygen (oxo, hydroxo, water, serine, aspartic acid), sulfur (cysteine), and selenium atoms (selenocysteine)
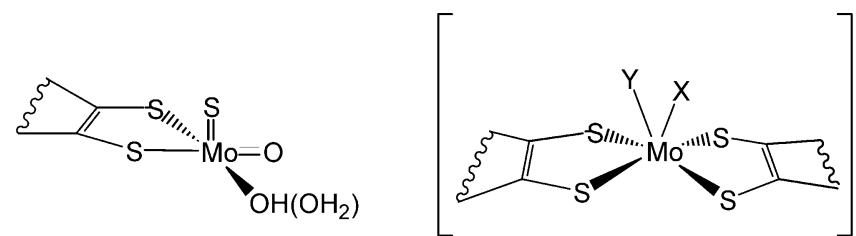

The Xanthine Oxidase Family

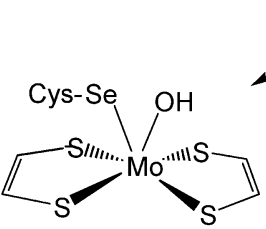

D. gigas $\mathrm{Fdh}(\mathrm{W})[18,19]$

E. coli Fdh-H [16]

E. coli Fdh-N [17]
The DMSO Reductase Family
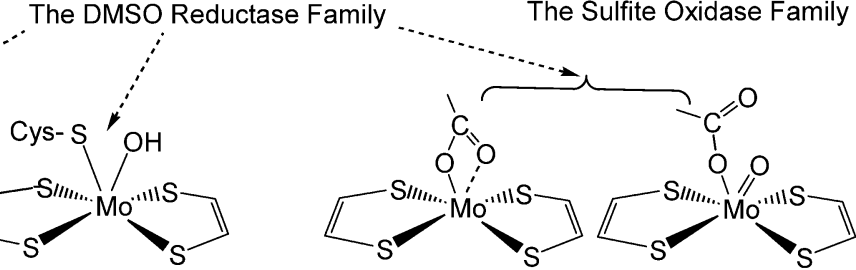

The Sulfite Oxidase Family

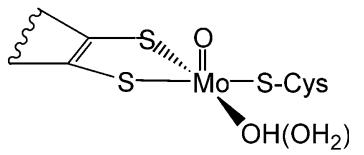

D. desulfuricans Nap[26] R. sphaeroides NapAB[27]
E coli NarGHI[24] pterin cofactor, and by a variable number of oxygen (oxo, hydroxo, water, serine, aspartic acid), sulfur (cysteines), and selenium atoms (selenocysteines). The pterin cofactor is an organic molecule which can have, in addition, a nucleotide molecule attached by a pyrophosphate link [9]. These proteins may also have other redox cofactors such as iron-sulfur centers, flavin, and hemes, which are assumed to be involved in electron transfer processes. This group of enzymes catalyzes, in general, the transfer of an oxygen atom from water to product (or vice versa) in reactions that imply a net exchange of two electrons between enzyme and substrate and in which the metal ion cycles between the redox states IV and VI. The members of the xanthine oxidase family catalyze the oxidative hydroxylation of a diverse range of aldehydes and aromatic heterocycles in reactions that involve the cleavage of a $\mathrm{C}-\mathrm{H}$ and the formation of a $\mathrm{C}-\mathrm{O}$ bond [10]. Mechanistic studies [11] and crystal structures of inhibited forms [12] of the enzymes indicate that the oxygen transferred to the substrate comes from the water/hydroxyl ligated to the Mo atom (Fig. 1). In contrast, the members of the other two families catalyze the transfer of an oxygen atom to or from a lone electron pair of the substrate, with the only exception of the formate dehydrogenases [13].

The proteins of the DMSO reductase family present the widest diversity of properties among members [1, 14]. The first crystal structure reported for a member of this family was DMSO reductase $[15,16]$. However, our knowledge about this family was greatly increased with the study of nitrate reductase, which catalyzes the reduction of nitrate to nitrite, and formate dehydrogenase, involved in the oxidation of formate to carbon dioxide. The metal atom in both enzymes is coordinated by two pterin cofactors, in which each pterin molecule has a guanine nucleotide, and different types of ligands identified as $\mathrm{X}$ and $\mathrm{Y}$ in Fig. 1. The few examples of enzymes capable of incorporating either molybdenum or tungsten at the active site belong to this family.

\section{Formate dehydrogenases: structure, function and spectroscopic properties}

Formate dehydrogenases (Fdh) catalyze the oxidation of formate to carbon dioxide according to the reaction:

$$
\mathrm{HCOO}^{-} \rightarrow \mathrm{CO}_{2}+\mathrm{H}^{+}+2 \mathrm{e}^{-} \quad E^{\circ}=-420 \mathrm{mV}
$$

The crystal structures of three Fdh proteins have been reported at present, two containing molybdenum and one tungsten. The Mo-containing enzymes are the Fdh$\mathrm{H}$ from Escherichia coli [17], which is one of the components of the formate hydrogen lyase complex in $E$. coli, and the recently reported membrane-bound Fdh-N from E. coli, which is supposed to participate in the redox loop of the proton motive force generation across the membrane cell [18]. The $\mathrm{W}$-containing enzyme is the Fdh from the sulfate-reducing bacterium (SRB) Desulfovibrio gigas $[19,20]$. E. coli $\mathrm{Fdh}-\mathrm{H}$ is a monomeric enzyme $(79 \mathrm{kDa})$, while the E. coli Fdh-N is an $\alpha \beta \gamma$ heterotrimer with subunits of 113,32 , and $21 \mathrm{kDa}$, respectively [18]. The W-Fdh from D. gigas is a heterodimeric enzyme with 92 and $29 \mathrm{kDa}$ subunits, respectively (Fig. 2a) [19, 20]. Most of the formate dehydrogenases characterized so far have a Se-cysteine residue bound to the Mo (or W). The structural data, in conjunction with spectroscopic data [12], suggest the direct involvement of the selenium cysteine Mo ligand and histidine 159 in proton abstraction by formate dehydrogenases (Fig. 2b). The overall structure of all three Fdh proteins is very similar: the superposition of the catalytic subunit of D. gigas W-Fdh with E. coli Fdh-H yields an r.m.s. deviation of $2.1 \AA$ for $659 \mathrm{C} \alpha$ atoms, while the structure of the $\alpha$-subunit of the E. coli Fdh-N is superimposable with that from $E$. coli $\mathrm{Fdh}-\mathrm{H}$ with an r.m.s. deviation of $1.9 \AA$ for $599 \mathrm{C} \alpha$ atoms.

Kinetic studies carried out with $E$. coli $\mathrm{Fdh}-\mathrm{H}$ showed that the oxidation of formate occurs via cleavage of the $\mathrm{C}-\mathrm{H}$ bond, resulting in a single $\mathrm{C}=\mathrm{O}$ bond [13]. This constitutes an exception for the general mechanism of 


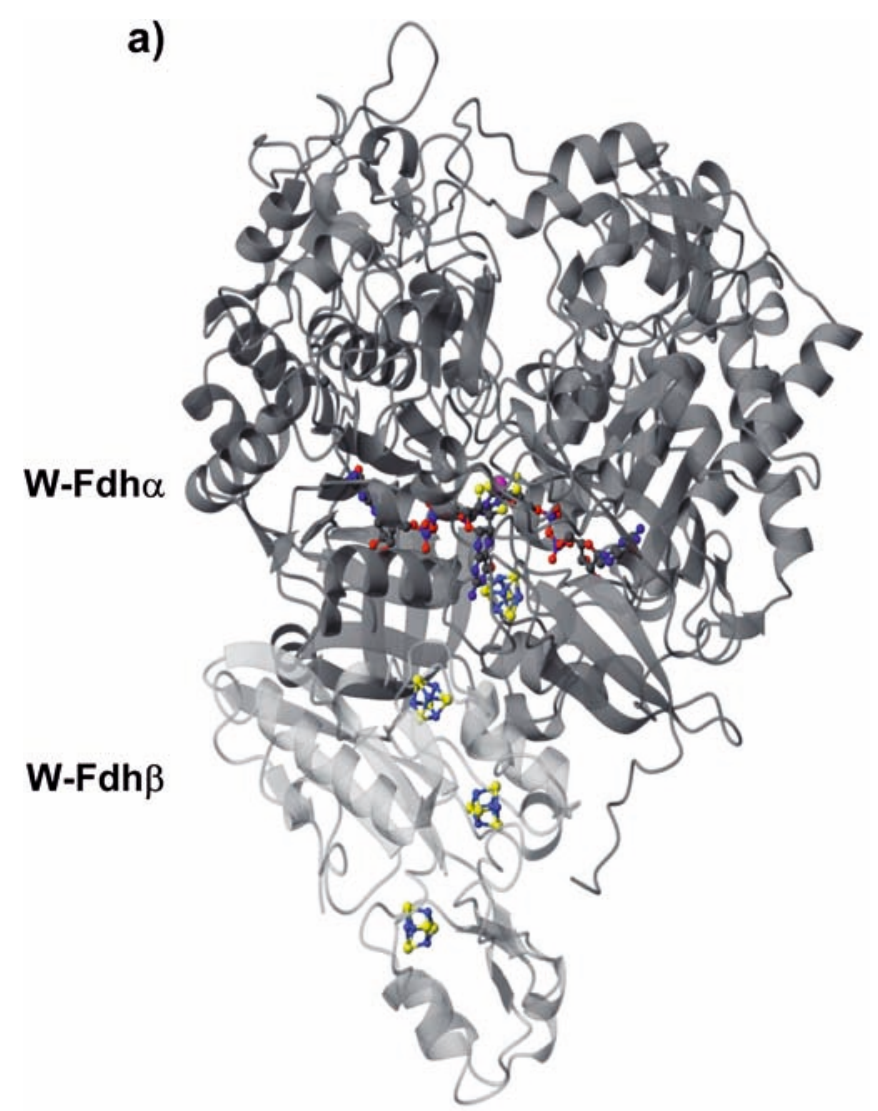

b)

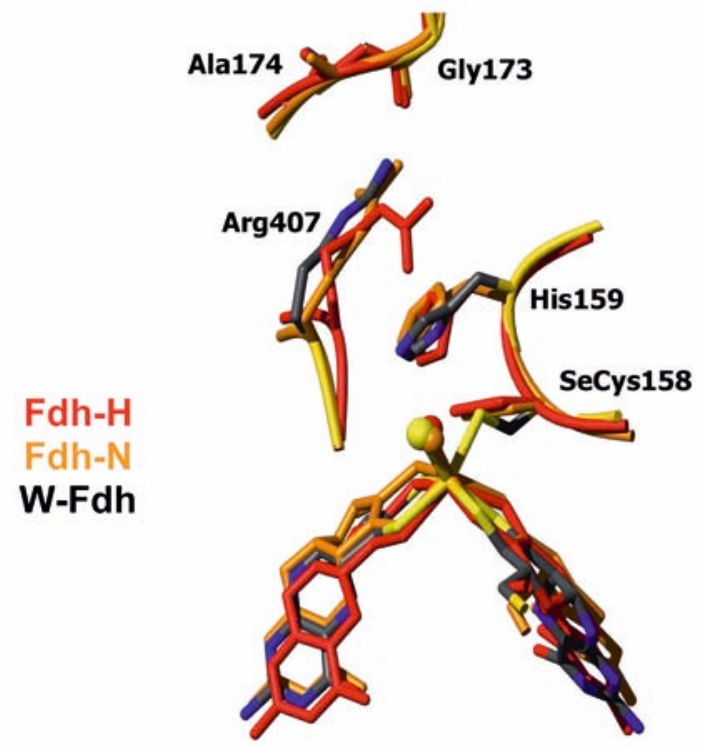

Fig. 2 (a) Overall structure of D. gigas W-Fdh. W-Fdh $\alpha$ is shown in dark gray, W-Fdh $\beta$ in light gray. The Mo-bis-MGD and the FeS clusters are represented as color-coded balls-and-sticks. (b) Comparison of the Fdh active sites. Superposition of the catalytic active sites of Fdh-H (red), Fdh-N (gold), and W-Fdh (color coded), evidencing the conserved residues important for the reaction. Residue numbering corresponds to W-Fdh

the mononuclear Mo- and W-containing enzymes, since these do not incorporate oxygen from water into the product. The current proposed mechanism of action for these enzymes suggests a replacement of the hydroxyl group bound to the molybdenum by one of the oxygen atoms from formate, followed by reduction of $\mathrm{Mo}(\mathrm{VI})$ to $\mathrm{Mo}(\mathrm{IV})$ and the cleavage of the $\mathrm{C}-\mathrm{H}$ bond [17]. However, Raaijmakers et al. [20] propose that the hydroxyl ligand (see Fig. 1) might be a sulfur atom in $D$. gigas Fdh, in which case the reaction mechanism should be re-evaluated. The inverse reaction, the conversion of $\mathrm{CO}_{2}$ to formate, has been demonstrated in a few cases, and this activity was found to be exclusive for W-Fdh proteins [4]. Consequently, it was suggested that the reduction of $\mathrm{CO}_{2}$ to formate is $\mathrm{W}$-dependent, whereas the inverse reaction in anaerobes is performed exclusively by Mo-containing enzymes [4]. Additional studies should be performed to verify whether the mechanism proposed for E. coli $\mathrm{Fdh}-\mathrm{H}$ is valid for all formate dehydrogenases [13] and if the conversion of $\mathrm{CO}_{2}$ to formate is exclusive for W-Fdh proteins [5].

Fdh from the SRB D. desulfuricans and D. alaskensis have been purified and characterized, and are closely related to D. gigas W-Fdh [23]. D. desulfuricans Fdh contains Mo at the active site $[21,22]$, but $D$. alaskensis Fdh can incorporate either Mo or W [23]. The Fdh from SRB present, in addition to the large subunit, a second subunit $(\sim 29 \mathrm{kDa})$, which was shown by X-ray studies of the D. gigas enzyme to contain four [4Fe-4S] clusters $[19,20]$. D. desulfuricans $\mathrm{Fdh}$ has also a third subunit (16 kDa), which contains four $c$-type hemes [21]. EPR and Mössbauer studies showed the presence of a variable number of $\mathrm{Fe} / \mathrm{S}$ clusters. All $\mathrm{Fdh}$ proteins from SRB exhibit an EPR signal that appears around $70 \mathrm{~K}$ and shows no broadening below $40 \mathrm{~K}$, usually named $\mathrm{Fe} / \mathrm{S} \mathrm{I}$ (mean $g$-values: $g_{1}=2.048, g_{2}=1.947, g_{3}=1.890$ ), which is similar to those observed in $D$. desulfuricans periplasmic nitrate reductase (Nap) [34] and in E. coli Fdh-H [13]. A second broader EPR signal (Fe/S II) appears below $30 \mathrm{~K}$ for all the members (mean $g$-values: $\left.g_{1}=2.067, g_{2}=1.921, g_{3}=1.873\right)$, and a third broad signal can be discernible in $D$. alaskensis Fdh below 20 K. D. alaskensis Fdh presents the Mo site magnetically coupled to one of the iron-sulfur clusters, a fact that was never seen before in proteins having a bis-pterin cofactor with a guanine dinucleotide (bis-MGD) coordination. The analysis of the inter-center magnetic interaction showed that $\mathrm{Fe} / \mathrm{S} \mathrm{I}$ is the center closest to the active site in Fdh from SRB [23].

\section{Nitrate reductases: structure, function and spectroscopic properties}

Nitrate reductases catalyze the reduction of nitrate to nitrite according to the reaction:

$$
\mathrm{NO}_{3}^{-}+2 \mathrm{H}^{+}+2 \mathrm{e}^{-} \rightarrow \mathrm{NO}_{2}^{-}+\mathrm{H}_{2} \mathrm{O} \quad E^{\circ}=420 \mathrm{mV}
$$

This reaction can be catalyzed by different enzymes, which can be distinguished by their taxonomy, function, location in the cell, structure, and the metabolic route in 
which they participate [24]. With the exception of the eukaryotic assimilatory nitrate reductases, which are part of the sulfite oxidase family [1], nitrate reductases belong to the DMSO reductase family of Mo-containing enzymes. The reduction of nitrate to nitrite by the Nap periplasmic pathway is considered to be a dissimilatory one, since it is not coupled to the generation of a proton gradient across the membrane. In contrast, the respiratory nitrate reduction pathway involves a membranebound protein complex composed of three subunits, NarGHI from E. coli $[25,26]$, which is involved in the redox loop of the proton motive force across biomembranes. NarG is the catalytic subunit and the whole complex is oriented to the cytoplasm.

\section{Periplasmic nitrate reductases}

Until now, only two crystal structures of periplasmic nitrate reductases have been determined: $D$. desulfuricans NapA (80 kDa) [27], which is the only monomeric Nap characterized so far, and the heterodimeric Rhodobacter sphaeroides NapAB [28]. The catalytic subunit of $R$. sphaeroides NapA (91 kDa) contains the bis-MGD cofactor and one [4Fe-4S] center. The smaller subunit NapB $(17 \mathrm{kDa})$ contains two $c$-type hemes and acts as an electron transfer subunit. Another protein involved is NapC, a membrane-bound multi-heme cytochrome $c$, which uses the quinol pool to provide electrons to the soluble complex NapAB.

The crystal structure of $D$. desulfuricans NapA was solved in 1999 at $1.9 \AA$ resolution [27] (see Fig. 3a). In 2002, Brige et al. [29] reported the high-resolution structure $(1.25 \AA)$ of a proteolytic fragment of the NapB subunit from Haemophilus influenzae, revealing the fold and heme arrangement. However, the crystal structure of the heterodimeric enzyme NapAB was determined only recently through the work of Arnoux et al. [28], with the structure of $R$. sphaeroides NapAB solved at $3.2 \AA$ resolution.

The $D$. desulfuricans Nap protein was isolated from the SRB D. desulfuricans ATCC 27774, which is able to grow on nitrate, inducing the enzymes responsible for the conversion of nitrate to nitrite and from nitrite to ammonia. The latter reaction involves a six-electron reduction and is catalyzed by a multiheme cytochrome $c$ type nitrite reductase (Nir) [30, 31]. The Nap molecule has an ellipsoidal shape with approximate dimensions of $65 \AA \times 65 \AA \times 50 \AA$ and its polypeptide fold is of the $\alpha / \beta$ type (Fig. 3a). It is organized into four domains (I-IV), three of which are formed by non-contiguous stretches of the polypeptide chain. All four domains are involved to variable extents in cofactor binding and, while the $[4 \mathrm{Fe}-4 \mathrm{~S}]$ center is located at the periphery of the molecule, the bis-MGD molybdenum cofactor extends across the interior of the molecule and interacts with residues from all four domains.

The spatial arrangement of domains II and III creates a funnel-like cavity at their interface, focused towards

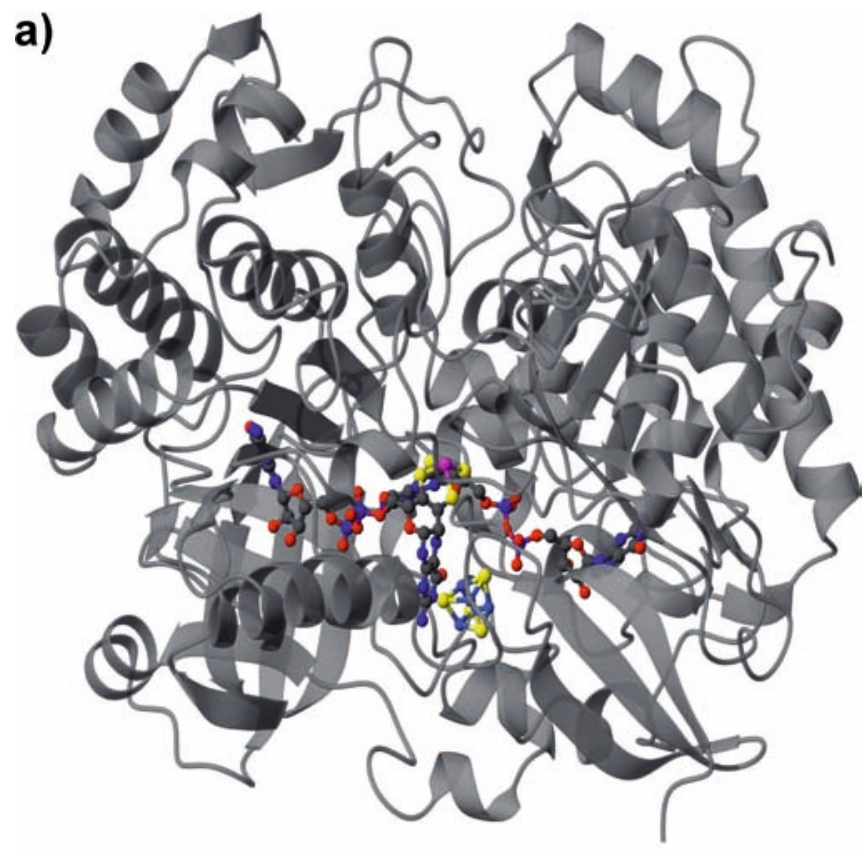

b)

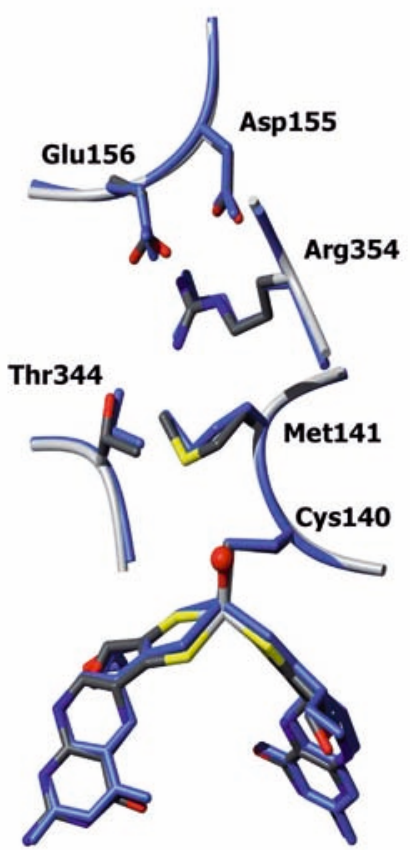

Fig. 3 (a) Overall structure of $D$. desulfuricans NapA. The Mo-bisMGD and FeS cluster are represented as color-coded balls-andsticks. (b) Superposition of the active sites of $D$. desulfuricans NapA (color coded) and $R$. sphaeroides NapA (blue). Residue numbering corresponds to $D$. desulfuricans NapA

the molybdenum center and located ca. $15 \AA$ below the molecular surface. The hydroxo/water ligand of the molybdenum points in the direction of this channel, which is coated with a few clustered charged residues (Arg354, Asp155, Glu156). Looking from the surface to the metal, Arg354 lies almost above the catalytic site 
(Fig. 3b) and might provide a suitable environment for binding nitrate.

The catalytic subunits of $D$. desulfuricans Nap and $R$. sphaeroides Nap are highly similar in terms of global fold and domain organization, with an r.m.s. deviation of only $1.26 \AA$ for $683 \mathrm{C} \alpha$ atoms (Fig. 3b). Their primary sequence identity is $35 \%$. The superposition of both structures reveals that the geometry at the bisMGD molybdenum active site, as well as the surrounding residues, is conserved. In the case of the $R$. sphaeroides Nap, the Mo active site was modeled on the basis of the $D$. desulfuricans NapA structure, due to the low resolution $(3.2 \AA)$ of the data.

Paracoccus denitrificans NapAB is closely related to $D$. desulfuricans and $R$. sphaeroides Nap proteins and constitutes the best spectroscopically characterized periplasmic nitrate reductase $[32,33]$. The EPR properties of this enzyme indicate a Mo site with a high flexibility of coordination, as shown by the different EPR-active species obtained with the enzymes poised at different redox potentials and in the presence of different inhibitors. The enzyme in the as-prepared form shows a $\mathrm{Mo}(\mathrm{V})$ EPR signal (high $g$ resting) typical of $\mathrm{Mo}(\mathrm{V})$ ion species $\left(g_{1}=1.998, g_{2}=1.990, g_{3}=1.981\right)$ split by two non-solvent-exchangeable species with nuclear spin $I=1 / 2$, presumed to be protons $\left(A_{\mathrm{av}}^{1}=5.5 \mathrm{G}\right.$, $\left.A^{2}{ }_{\mathrm{av}}=2.8 \mathrm{G}\right)$ [32]. A similar resting EPR signal was detected in $R$. sphaeroides NapAB [28]. With the enzyme in turnover conditions, the resting signal is replaced with a new $\operatorname{Mo}(\mathrm{V})$ ion signal (high $g$ nitrate) showing a hyperfine split with a single non-solvent-exchangeable proton $\left(g_{1}=1.999, g_{2}=1.989, g_{3}=1.981, A_{\mathrm{av}}=5.1 \mathrm{G}\right)$, which was suggested to be a catalytic intermediate. This signal is identical to that obtained in $D$. desulfuricans Nap when reduced samples are reoxidized with nitrate [34]. These preliminary data obtained in Nap from $D$. desulfuricans and $R$. sphaeroides suggest that the active sites of the three enzymes are similar. This is in contradiction to the structure deduced from extended X-ray absorption fine structure (EXAFS) studies, which indicates that besides the bi-pterin coordination, the active site presents three extra ligands [32]. Additional work should be performed to clarify these discrepancies.

\section{Membrane-bound nitrate reductases}

Recently, two independent research groups solved the three-dimensional structure of the cytoplasmatic, membrane-bound nitrate reductase from E. coli. In 2003 the whole complex NarGHI was reported, at a resolution of $1.9 \AA$ [25], and in 2004 the heterodimer NarGH was solved to $2.0 \AA$ resolution [26]. The heterotrimer NarGHI comprises a catalytic subunit (NarG, $140 \mathrm{kDa})$, a subunit containing four $[4 \mathrm{Fe}-4 \mathrm{~S}]$ centers $(\mathrm{NarH}$, $58 \mathrm{kDa}$ ), and a membrane-bound heme $b$ subunit (NarI, $20 \mathrm{kDa}$ ) (Fig. 4a).

Several novel aspects were revealed by these two Nar structures. (1) For the first time, the carboxylate from an aspartate (Asp222) side chain is coordinating the Mo but showing different coordination modes (see below). (2) In the NarGHI structure, one of the MGD cofactors (MGD-Q) is present in a bicyclic dihydropterin structure (Fig. 4b), rather than the tricyclic pyranopterin structure found so far in all Mo enzymes. (3) In addition, the [4Fe$4 \mathrm{~S}$ ] cluster present in the catalytic subunit (FeS-0) has an unusual coordination by one histidine and three cysteines (His50, Cys54, Cys58, Cys93), which had only been found in D. gigas hydrogenase [35].

The NarG global architecture and domain organization is similar to the related Nap or Fdh structures. However, in the region close to the active site, as well as in the tunnel that connects the Mo site to the exterior of the protein, there are several differences that include unexpected features. The Mo atom is coordinated by two dithiolene sulfur atoms from both MGD cofactors as well as to the carboxylate of an aspartate residue, Asp222, which is highly conserved (Figs. 1 and $4 \mathrm{~b}$ ). This is the first time that an Asp-Mo coordination is observed in bis-MGD enzymes. In addition, while the carboxylate group acts as a bidentate ligand in the complete NarGHI structure (distances of $\sim 1.9 \AA$ and $\sim 2.4 \AA$ for Mo-OD1 and Mo-OD2, respectively) [25], in the NarGH structure Asp222 makes only one bond to the Mo atom [26]. The sixth coordination position is occupied by an oxo group $(\mathrm{Mo}=\mathrm{O} \approx 1.8 \AA$ ) which is absent in the NarGHI structure. In both cases the crystals were grown aerobically and therefore both structures must represent the oxidized form of the enzyme. The coordination of metal sites by side-chain carboxylates from aspartic and glutamic acid molecules has been the subject of several studies ([36] and references therein). These studies showed that carboxylate groups present a high flexibility of coordination, as suggested by Jormakka et al. [26].

In addition, the structure with the bidentate binding of Asp222 reveals an open bicyclic form of the pterin (MGDQ) (Fig. 4b), in contrast to all crystal structures published so far, which show the pterin cofactor in the tricyclic pyranopterin form as found in NarGH. The comparison of both structures reveals that in the bicyclic structure the free hydroxyl group at $\mathrm{C} 2$ is stabilized by hydrogen bonding interactions to Ser719 and to His1163 (Fig. 4b). In the tricyclic structure the side chain of Ser719 and the corresponding loop adopt a different conformation. Redox reactivity studies of the pterin cofactor were recently reported by Burgmayer et al. [37] and have shed light on the reactivity aspects of the isolated pterin cofactor. These studies, together with the two different interconvertible forms of the cofactor present in the E. coli NarG structures, are a starting point towards a better understanding of the true role of the pterin cofactor in the enzymatic mechanism that occurs during catalysis in Mo- and Wcontaining enzymes.

The redox and EPR properties of the active site of membrane Nar obtained from different sources has been the subject of several studies [38, 39, 40, 41, 42, 43]. Nar enzymes show $\mathrm{pH}$-dependent $\mathrm{Mo}(\mathrm{V})$ ion EPR signals having resonances split by a solvent-exchangeable pro- 
Fig. 4 (a) Overall structure of NarGHI. NarG is represented in dark gray, $\mathrm{NarH}$ in gray, and the membrane-bound NarI in light gray. The Mo-bis-MGD, FeS clusters, and heme groups are represented as color-coded balls-and-sticks. (b) Top: the Nar Mo-bis-MGD cofactor. Comparison of the bicyclic and tricyclic forms of the pterin (MGD-Q) found in the two structures of NarG. Asp222 switches between a mono- and bidentate coordinator of the Mo atom. Ser719 and His1163 help stabilize the open pterin form. NarGHI is shown colorcoded and NarGH is light yellow. Bottom: schematic representation of the interconversion between the open and closed forms of the pterin

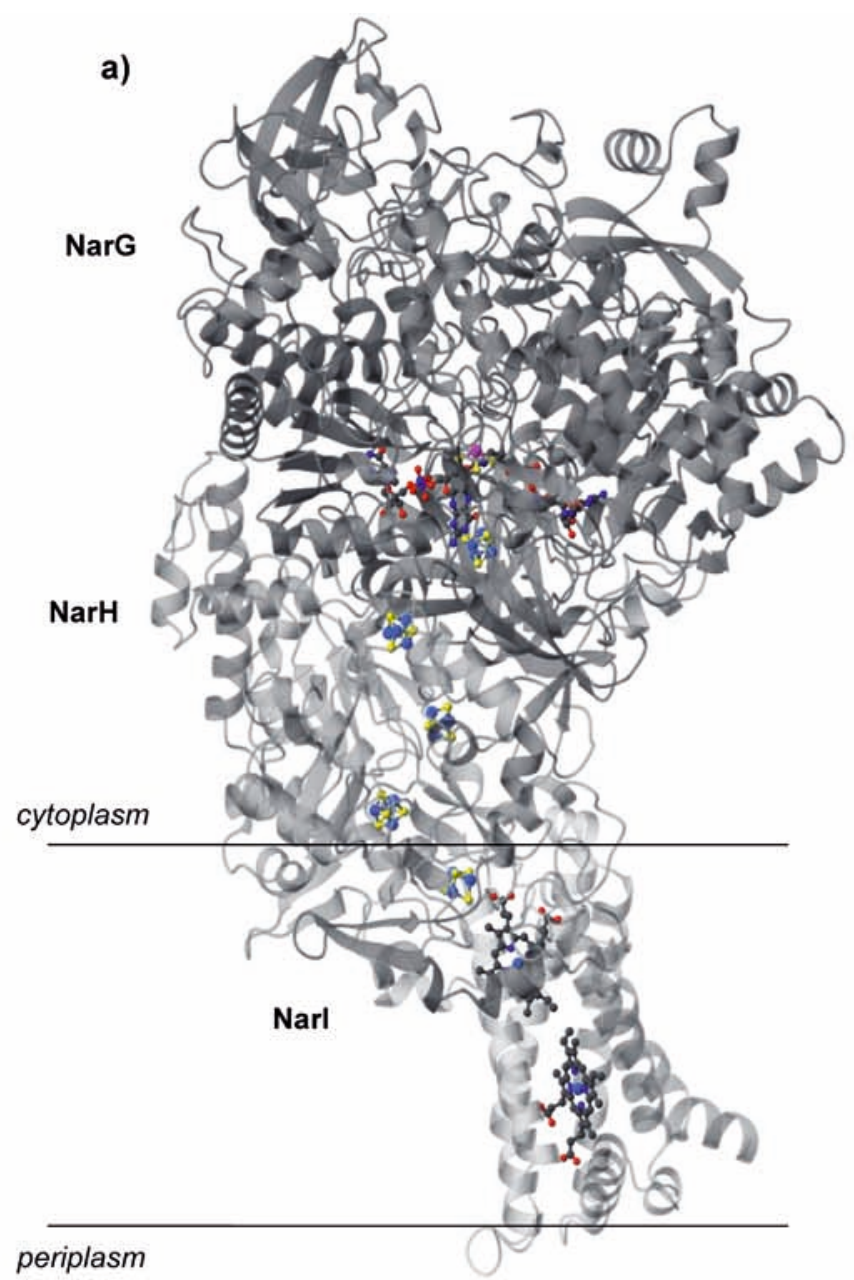

b)

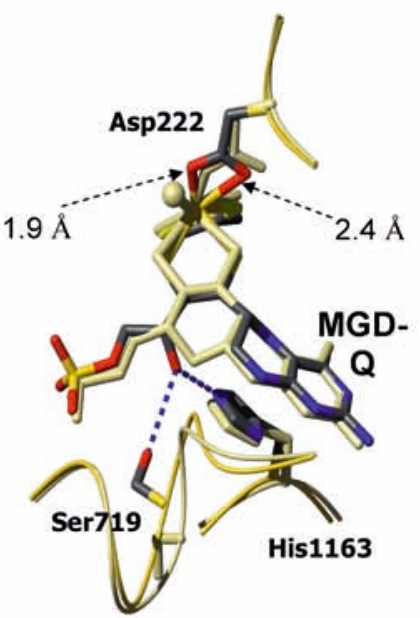

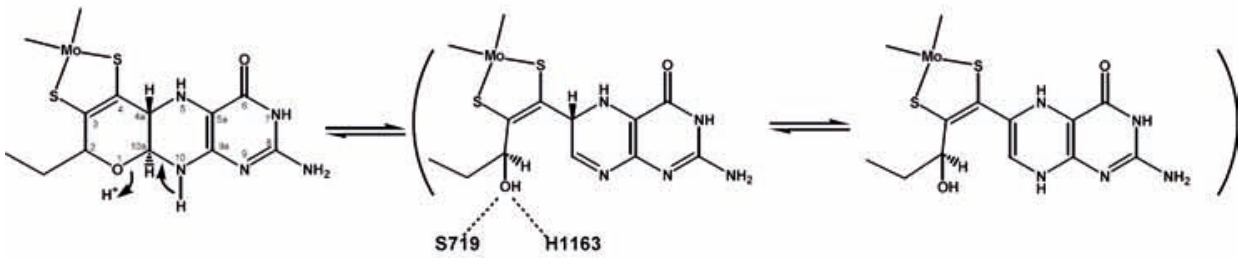

Nar GH

NarGHI 
ton. The EPR-active species are identified as low-pH $\left(g_{1}=2.001, g_{2}=1.986, g_{3}=1.966, A_{\mathrm{av}}=9.3 \mathrm{G}\right)$ and high $\mathrm{pH}\left(g_{1}=1.987, g_{2}=1.981, g_{3}=1.962, A_{\mathrm{av}}=3.4 \mathrm{G}\right)[39]$. The catalytic role of these species is still in debate. The molecular structure for the active site in the $\mathrm{Mo}(\mathrm{VI})$ redox state reported for NarGHI [25] does not depict a water/hydroxyl ligand, as expected from the EPR data. However, the structure of NarGH shows that the bidentate coordination of the aspartic acid is opened, allowing the coordination of an oxo ligand. This suggests that this ligand might be the entry site for the substrate, as suggested for formate dehydrogenases (see above). EPR studies of E. coli Nar detected the presence of four $\mathrm{Fe} / \mathrm{S}$ clusters in the NarH subunit but did not detect the $\mathrm{Fe} / \mathrm{S}$ center in the NarG subunit $[42,43]$. EPR studies performed in E. coli clearly showed the typical resonances for the $3 \mathrm{Fe}-4 \mathrm{~S}$ cluster and two EPR signals, which were attributed to two different conformations of a single $\mathrm{Fe} / \mathrm{S}$ cluster, but the EPR properties of the remaining $\mathrm{Fe} / \mathrm{S}$ centers could not be determined precisely. Jormakka et al. [26] hypothesized that the $\mathrm{Fe} / \mathrm{S}$ cluster in the NarG subunit should be both a high-redox potential center, because of the polar environment around it, and a high-spin center, to explain the nondetection of this center by EPR. However, as seen below, [4Fe-4S] centers with a similar environment but with low redox potentials were found in all the proteins described in this review. Clearly, additional work should be performed to confirm this hypothesis and also to assign the EPR signals associated with the [4Fe-4S] clusters to those seen in the crystal structure.

All the redox cofactors in E. coli Nar are along a chemical path for electron transfer, but the redox potentials evaluated for two of the iron-sulfur clusters show redox potentials that do not favor electron transfer. Therefore, it was suggested that the iron-sulfur center with a positive redox potential should vary during turnover conditions to obtain an efficient electron transfer path [26, 44]. This possibility was recently demonstrated in a copper-containing nitrite reductase from Pseudomonas chlororaphis DSM 50135, in which the binding of the substrate to the active center modulates the rate of intramolecular electron transfer, changing the values of the redox potentials of the redox centers [45]. Electrochemical studies on $R$. sphaeroides Nap show that the kinetics of substrate binding favors intramolecular electron transfer [46]. Furthermore, redox potentials alone cannot be used to predict electron transfer in biological systems and additional factors must be considered [47].

\section{Comparison of nitrate reductases and formate dehydrogenases}

The similarity of the overall 3D structures is particularly high between the periplasmic nitrate reductases and formate dehydrogenases. The superposition of the large subunit of $D$. gigas W-FDH with $D$. desufuricans NapA shows an r.m.s. deviation of $2.0 \AA$ for $636 \mathrm{C} \alpha$ atoms, while the comparison with $E$. coli FDH-H gives a corresponding r.m.s. deviation of $2.1 \AA$ for $659 \mathrm{C} \alpha$ atoms. The structure of the $\alpha$-subunit of the E. coli Fdh-N is superimposable with that from $E$. coli Fdh-H with an r.m.s. deviation of $1.9 \AA$ for $599 \mathrm{C} \alpha$ atoms. In the larger proteins, E. coli Fdh-N and D. gigas W-FDH, the additional residues of the catalytic $\alpha$-subunit are essentially distributed over the molecular surface as insertions in the whole polypeptide. In the vicinity of the active sites there are clear differences in the amino acid distribution for both types of enzymes (Fig. 5), which must account for their different specificities.

\section{Electron transfer pathway in nitrate reductases and formate dehydrogenases}

The electron transport pathway between the Mo active site and an external physiological electron acceptor (or donor) is similar in both enzymes. It has been assumed [17] that in formate dehydrogenases and in periplasmic nitrate reductases a conserved Lys residue mediates the electron transfer between one of the pterin cofactors and the relatively exposed [4Fe-4S] cluster (Fig. 6a). The important

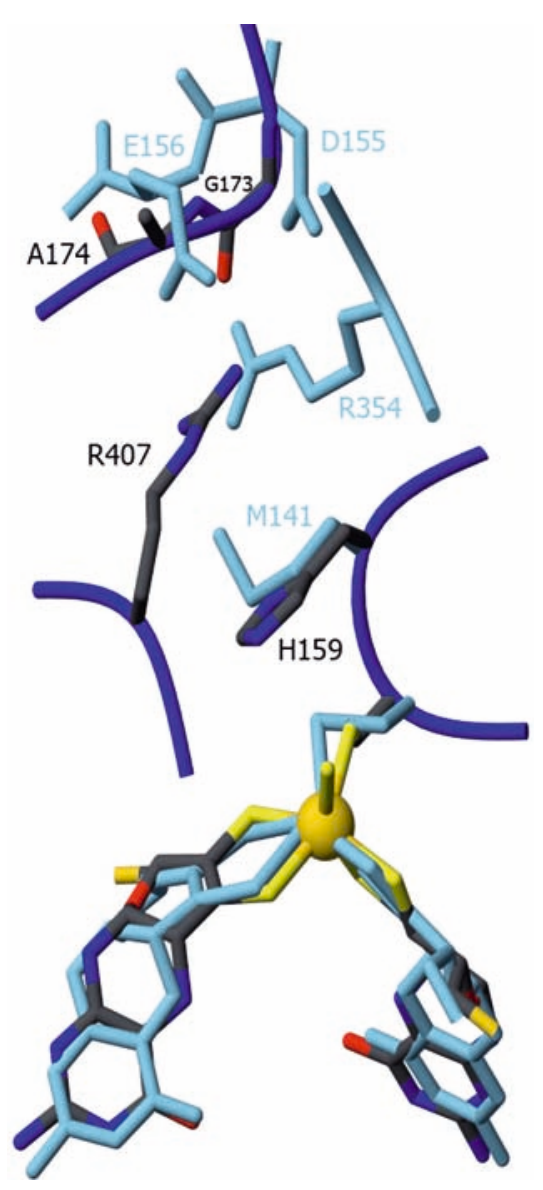

Fig. 5 Comparison of the Nap and Fdh active sites. Superposition of W-Fdh (color-coded) and NapA (light blue). Residues surrounding the active site are represented as sticks, with the respective labels in black (W-Fdh) and blue (NapA) 
role of this lysine residue has been shown by mutagenesis studies of NapAB from Ralstonia eutropha [48]. The mutant K85R showed $23 \%$ of the wild-type activity, while $\mathrm{K} 85 \mathrm{M}$ resulted in total loss of activity, suggesting the requirement of a positively charged residue for an effective electron transport between both metallic centers.

In the case of Nar, they carry a conserved Arg residue at the corresponding position of Lys [49]. This arginine residue is close to the $\mathrm{Fe} / \mathrm{S}$ cluster present in the NarG subunit and is also in close proximity to the exocyclic $\mathrm{NH}_{2}$ of the pterin ring of MGD-P (Fig. 6b). Its role may also be to mediate the electron transfer between the

a)

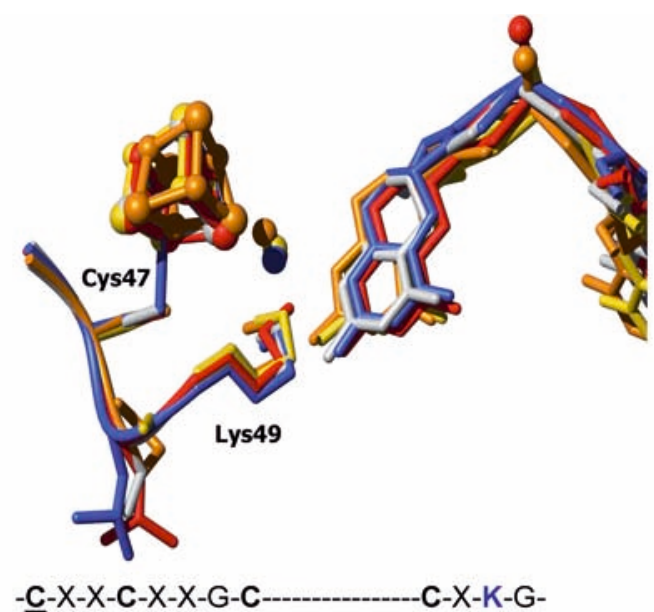

b)

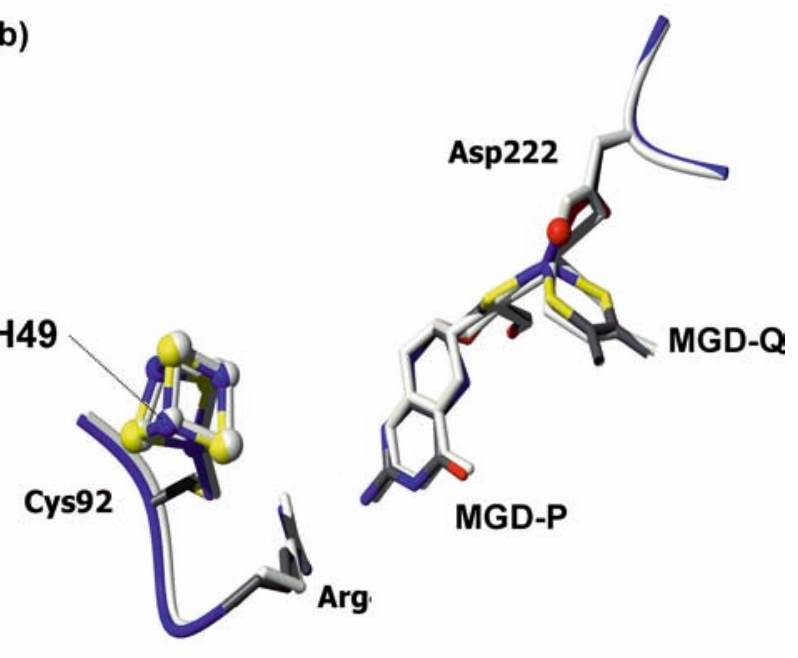

$-\mathrm{H}^{49}-X-X-X-C^{53}-X-X-X-C^{57}$

Fig. 6 Electron transfer pathways in the catalytic subunits of Nap, Fdh, and Nar. (a) Superposition of the molybdopterin and $\mathrm{Fe} / \mathrm{S}$ cluster of all the structures of Nap and Fdh available. D. desulfuricans NapA is shown in blue, $R$. sphaeroides NapAB in gray, E. coli $\mathrm{Fdh}-\mathrm{H}$ in red, E. coli $\mathrm{Fdh}-\mathrm{N}$ in gold, and D. gigas WFdh in yellow. Lys49 (in D. desulfuricans NapA) and a conserved water molecule involved in the electron transfer pathway are also represented. The guanine nucleotide was removed from the bisMGD for clarity. (b) Superposition of molybdopterin and FeS-0 of the Nar structures, evidencing Arg94 as possibly involved in the electron transfer pathway. NarGH is color-coded and NarGHI is gray redox centers or to modulate the redox potential of the FeS- 0 center.

\section{Incorporation of either Mo or W in enzymes}

The analogous chemical properties of $\mathrm{W}$ and Mo and the similar active sites of $\mathrm{W}$ - and Mo-containing enzymes led us to assume that $\mathrm{W}$ could replace Mo in enzymes. Though the first intents of substituting $\mathrm{W}$ for Mo only yielded either metal-free inactive enzymes or W-substituted enzymes with insignificant or no activity [2], recently it was shown that the Mo enzymes $R$. capsulatus DMSO reductase [50] and E. coli trimethylamine $N$-oxide (TMAO) reductase [51] can incorporate $\mathrm{W}$ and keep enzyme activity. More recently, the characterization of the enzyme $D$. alaskensis $\mathrm{Fdh}$, which can incorporate Mo and $\mathrm{W}$ at the active site, was reported [23]. $R$. capsulatus W-DMSO reductase and E. coli W-TMAO reductase were obtained in a medium supplemented with tungsten, but $D$. alaskensis $\mathrm{W}-\mathrm{Fdh}$ and Mo-Fdh were co-purified in a medium with traces of $\mathrm{W}$ and Mo, which was shown to better mimic the growth conditions experienced by SRB in their natural habitats [52].

The question of why some enzymes can function with either metal ion while others seem to be exclusive for one is far from being elucidated and, at this stage, one can only give speculative reasons. These can be, apparently, divided into (1) redox properties of the enzyme, (2) bioavailability of the metal ions, and (3) evolution.

1. Redox properties. W-containing enzymes generally catalyze reactions with low redox potentials $(\leq-420 \mathrm{mV})[2,6]$. In the case of W-Fdh enzymes, these were also shown to catalyze the conversion of $\mathrm{CO}_{2}$ to formate [5], a fact not yet demonstrated in any Mo-Fdh. This is consistent with the fact that $\mathrm{W}(\mathrm{IV})$ is a stronger reducer than Mo(IV). However, a $\mathrm{W}$ enzyme catalyzing a reaction with positive redox potentials was observed [51].

2. Bioavailability. This seems to be the case for some enzymes but not for others. For instance, $R$. capsulatus DMSO reductase and E. coli TMAO reductase incorporate and function with $\mathrm{W}$ when this metal is more available in the medium. In the same line, $D$. alaskensis $\mathrm{Fdh}$ can incorporate both metal ions in a medium with traces of both metals. In the same medium where $D$. alaskensis $\mathrm{Fdh}$ was obtained, an aldehyde oxidoreductase (AOR), almost identical to the Mo-AORs from SRB, contains only Mo [53]. In contrast, D. gigas Fdh [3] and Fdh enzymes from Syntrophobacter fumaroxidans incorporate W [5], despite their growth with enough Mo in the medium. These examples suggest that bioavailability is not the only condition for an enzyme to incorporate either Mo or $\mathrm{W}$ and that some enzymes can function only with a specific ion, as in the case of the W-Fdh described above, the $\mathrm{W}$ enzymes found in hyperthermofilic archaea [2, 6], and the Mo-AOR from SRB. 
3. Evolution. Different authors seem to agree that Wcontaining isoenzymes represent, apparently, less evolved forms or ancestors of the Mo-containing enzymes $[3,5,51]$. In this context, the existing Wcontaining enzymes, such as $D$. gigas and $S$. fumaroxidans Fdhs [3, 4], would represent a group of enzymes that cannot adopt Mo to accomplish a specific function in living organisms, which along evolution were substituting Mo for $\mathrm{W}$ in enzymes.

The incorporation of either Mo or $\mathrm{W}$ in enzymes is far from being elucidated, and additional work in the existing systems together with the characterization of new enzymes that can function with both metal ions might be useful to elucidate the evolutionary relationships of the Mo- and W-containing enzymes.

PDB codes of enzymes mentioned in this review: $D$. desulfuricans NapA, 2NAP; D. gigas W-Fdh, $1 \mathrm{H} 0 \mathrm{H} ; E$. coli $\mathrm{Fdh}-\mathrm{H}, 1 \mathrm{FDO}$; E. coli $\mathrm{Fdh}-\mathrm{N}, 1 \mathrm{KQF}$; E. coli NarGH, 1R27; E. coli NarGHI, 1Q16; $R$. sphaeroides Na$\mathrm{pAB}, 1 \mathrm{OGY}$.

Acknowledgements C.D.B. and J.J.G.M. thank SECYT (Argentina) and GRICES (Portugal) for a bi-national grant. The work was supported by projects EC HPRN-CT-1999-00084, POCTI/1999/ $\mathrm{BME} / 35078$, and POCT/BME/36152/99.

\section{References}

1. Hille R (1996) Chem Rev 96:2757-2816

2. Johnson MK, Rees DC, Adams MW (1996) Chem Rev 96:2817-2840

3. Almendra MJ, Brondino CD, Gavel O, Pereira AS, Tavares P, Bursakov S, Duarte R, Caldeira J, Moura JJ, Moura I (1999) Biochemistry 38:16366-16372

4. Hensgens CMH, Hagen WR, Hansen TH (1995) J Bacteriol 177:6195-6200

5. de Bok FAM, Hagedoorn PL, Silva PJ, Hagen WR, Schiltz E, Fritsche K, Stams E, Fritsche K, Stams AJM (2003) Eur J Biochem 270:2476-2485

6. Hagen WR, Arendsen AF (1998) Struct Bonding 90:161-192

7. Kisker C, Schindelin H, Rees DC (1997) Annu Rev Biochem 66:233-267

8. Romao MJ, Knablein J, Huber R, Moura JJ (1997) Prog Biophys Mol Biol 68:121-144

9. Stiefel EI (1997) J Chem Soc Dalton Trans 3915-3923

10. Romao MJ, Cunha CA, Brondino CD, Moura JJ (2002) Met Ions Biol Syst 39:539-570

11. Xia M, Dempski R, Hille R (1999) J Biol Chem 274:3323-3330

12. Truglio JJ, Theis K, Leimkuhler S, Rappa R, Rajagopalan KV, Kisker C (2002) Structure 10:115-125

13. Khangulov SV, Gladyshev VN, Dismukes GC, Stadtman TC (1998) Biochemistry 37:3518-3528

14. Basu P, Stolz JF, Smith T (2003) Curr Sci 84:1412-1418

15. Schindelin H, Kisker C, Hilton J, Rajagopalan KV, Rees DC (1996) Science 272:1615-1621

16. Schneider F, Löwe J, Huber R, Schindelin H, Kisker C, Knäblein J (1996) J Mol Biol 263:53-69

17. Boyington JC, Gladyshev VN, Khangulov SV, Stadtman TC, Sun PD (1997) Science 275:1305-1308

18. Jormakka M, Tornroth S, Byrne B, Iwata S (2002) Science 295:1863-1868

19. Raaijmakers H, Teixeira S, Dias JM, Almendra MJ, Brondino CD, Moura I, Moura JJ, Romao MJ (2001) J Biol Inorg Chem $6: 398-404$
20. Raaijmakers H, Macieira S, Dias JM, Teixeira S, Bursakov S, Huber R, Moura JJ, Moura I, Romao MJ (2002) Structure 10:1261-1272

21. Costa C, Teixeira M, LeGall J, Moura JJG, Moura I (1997) J Biol Inorg Chem 2:198-208

22. George GN, Costa C, Moura JJG, Moura I (1999) J Am Chem Soc 121:2625-2626

23. Brondino CD, Passeggi MC, Caldeira J, Almendra MJ, Feio MJ, Moura JJ, Moura I (2004) J Biol Inorg Chem 9:145-151

24. Stolz JF, Basu P (2002) ChemBioChem 3:198-206

25. Bertero MG, Rothery RA, Palak M, Hou C, Lim D, Blasco F, Weiner JH, Strynadka NC (2003) Nat Struct Biol 10:681-687

26. Jormakka M, Richardson D, Byrne B, Iwata S (2004) Structure 12:95-104

27. Dias JM, Than ME, Humm A, Huber R, Bourenkov GP, Bartunik HD, Bursakov S, Calvete J, Caldeira J, Carneiro C, Moura JJ, Moura I, Romao MJ (1999) Structure 7:65-79

28. Arnoux P, Sabaty M, Alric J, Frangioni B, Guigliarelli B, Adriano JM, Pignol D (2003) Nat Struct Biol 10:928-34

29. Brige A, Leys D, Meyer TE, Cusanovich MA, Van Beeumen JJ (2002) Biochemistry 41:4827-4836

30. Almeida MG, Macieira S, Gonçalves LL, Huber R, Cunha CA, Romão MJ, Lampreia J, Moura JJG, Moura I (2003) Eur J Biochem 270:3904-3915

31. Cunha A, Macieira S, Dias JM, Almeida G, Gonçalves LL, Costa C, Lampreia J, Huber R, Moura JJG, Moura I, Romão MJ (2003) J Biol Chem 278:17455-17465

32. Butler CS, Charnock JM, Bennett B, Sears HJ, Reilly AJ, Ferguson SJ, Garner CD, Lowe DJ, Thomson AJ, Berks BC, Richardson DJ (1999) Biochemistry 38:9000-9012

33. Butler CS, Fairhurst SA, Ferguson SJ, Thomson AJ, Berks BC, Richardson DJ, Lowe DJ (2002) Biochem J 363:817-23

34. Bursakov SA, Liu MY, Payne WJ, LeGall J, Moura I, Moura JJG (1995) Anaerobe 1:55-60

35. Volbeda A, Charon MH, Piras C, Hatchikian EC, Frey M, Fontecilla-Camps JC (1995) Nature 373:580-587

36. Brondino CD, Calvo R, Atria AM, Spodine E, Nascimento OR, Pena O (1997) Inorg Chem 36:3183-3189

37. Burgmayer SJN, Pearsall DL, Blaney SM, Eva M, Moore EM, Sauk-Schubert C (2004) J Biol Inorg Chem 9:59-66

38. Vincent SP, Bray RC (1978) Biochem J 171:639-647

39. George GN, Bray RC, Morphet FF, Boxer DH (1985) Biochem J 227:925-931

40. Godfrey C, Greenwood C, Thomson AJ, Bray RC, George GN (1984) Biochem J 224:601-608

41. Hettmann T, Anemuller S, Borcherding H, Mathé L, Steinrucke P, Diekmann S (2003) FEBS Lett 534:143-150

42. Magalon A, Asso M, Guigliarelli B, Rothery RA, Bertrand P, Giordano G, Blasco F (1998) Biochemistry 37:7363-7370

43. Blasco F, Guigliarelli B, Magalon A, Asso M, Giordano G, Rothery RA (2001) Cell Mol Life Sci 58:179-193

44. Richardson D, Sawers G (2002) Science 295:1842-1843

45. Pinho D, Besson S., Brondino CD, de Castro B, Moura I (2004) Eur J Biochem 271:2361-2369

46. Frangioni B, Arnoux P, Sabaty M, Pignol D, Bertrand P, Guigliarelli B, Leger C (2004) J Am Chem Soc 126:1328-1329

47. Beratan DN, Onuchic JN, Winkler JR, Gray HB (1992) Science 258:1740-1741

48. Hettmann T, Siddiqui RA, von Langen J, Frey C, Romao MJ, Diekmann S (2003) Biochem Biophys Res Commun 310:40-47

49. Philippot L, Hojberg O (1999) Biochim Biophys Acta 1446:123

50. Stewart LJ, Bailey S, Bennett B, Charnock JM, Garner CD, McAlpine AS (2000) J Mol Biol 299:593-600

51. Buc J, Santini CL, Giordani R, Czjzek M, Wu LF, Giordano G (1999) Mol Microbiol 32:159-168

52. Feio MJ, Zinkevich V, Beech IB, Llobet-Brossa E, Eaton P, Schmitt J, Guezennec J (2004) Int J Syst Evol Microbiol (in press); http://dx.doi.org/10.1099/ijs.0.63118-0

53. Andrade SL, Brondino CD, Feio MJ, Moura I, Moura JJ (2000) Eur J Biochem 267:2054-2061 